CRISPR-Cas13a family and fourteen members of the CRISPRCas13b family.

We next focused on tuning the output of the SHERLOCK signal to make it more quantitative, sensitive, and robust to broaden the utility of the technology, testing optimizations of the amplification to enable quantitative and able to accept larger input volumes for increased sensitivity.

Another goal of SHERLOCKv2 was engineering a visual readout of activity requiring no additional instrumentation. We designed a lateral-flow readout that was based on the destruction of a FAM-biotin reporter, allowing for detection on commercial lateral flow strips. Abundant reporter accumulates anti-FAM antibody-gold nanoparticle conjugates at the first line on the strip, preventing binding of the antibody-gold conjugates to protein A on the second line; cleavage of reporter would reduce accumulation at the first line and result in signal on the second line. We profiled Cas13 cleavage preferences on homopolymer reporters, and found that most orthologs preferred either uridine, a combination of bases, or adenine. We refined the cleavage sequence preferences by evaluating collateral activity across di-nucleotide motifs, finding a large diversity of di-nucleotide cleavage motif preferences. ). From these di-nucleotide cleavage screens, we found that the activities of LwaCas13a, CcaCas13b, LbaCas13a and PsmCas13b could all be independently measured with the four di-nucleotide reporters AU, UC, AC, and GA, respectively. Additionally, using a random in vitro RNA library motif cleavage screen, we identified numerous RNA 6-mers that allowed for further orthogonality between Cas13 enzymes. When combined with RPA, we detected two DNA targets (the P. aeruginosa acyltransferase gene and the $S$. aureus thermonuclease gene) down to the attomolar range. These advances in in-sample multiplexing via orthogonal base preferences allow for many targets to be detected at scale and for cheaper cost.

We tested a range of primer concentrations and found that $240 \mathrm{nM}$ exhibited the greatest correlation between signal and input, and quantification was sustainable across a large range of sample concentrations down to the attomolar range. By scaling up the pre-amplification RPA step, we found that LwaCas13a could give detection signal for 200, 80, and 8zM input samples and allow for single-molecule volume inputs of $250 \mu \mathrm{L}$ and $540 \mu \mathrm{L}$.

We tested lateral flow with Cas13 for instrument-free detection of ZIKV or DENV ssRNA, and found that detection was possible in under 90 minutes with sensitivities down to the 2 aM condition. Moreover, we found that we could do rapid genomic DNA extraction from human saliva $(<10 \mathrm{~min})$ and input this directly into SHERLOCK without purification for rapid genotyping in under 23 minutes by fluorescence and 2 hours by lateral flow. The additional refinements presented here for Cas13-based detection allow for quantitative, visual, more sensitive, and multiplexed readouts, enabling additional applications for nucleic acid detection, especially in settings where portable and instrument-free analysis are necessary. SHERLOCKv2 can be used for multiplexed genotyping to inform pharmacogenomic therapeutic development and application, detecting genetically modified organisms in the field, or determining the presence of co-occurring pathogens. Moreover, the rapid, isothermal readout of SHERLOCKv2, enabled by lateral flow and Csm6, provides an opportunity for detection in settings where power or portable readers are unavailable, even for rare species like circulating DNA. In the future, it might be possible to make solution-based colorimetric readouts and multiplex lateral flow assays containing multiple test strips for different targets. Improved CRISPR-dx nucleic acid tests make it easier to detect the presence of nucleic acids in a range of applications across biotechnology and health and are now field-ready for rapid and portable deployment.

Disclosure No significant relationships.

\section{S15.3 REDUCING THE GLOBAL BURDEN OF INFECTIOUS DISEASES THROUGH PRECISION INFECTION MANAGEMENT (PIM)}

Ian Lewis*. University of Calgary, Biological Science, Calgary, Canada

10.1136/sextrans-2019-sti.71

The global rise in the prevalence of antibiotic resistant bacteria is a problem so serious that it threatens all modern medicine. One major factor contributing to the problem is the limited information available to clinicians regarding the risks posed by individual strains of pathogens. Titrating clinical interventions according to these risks would enable the more judicious use of antibiotics, would reduce the time necessary to control serious infections, and would minimize antibioticassociated treatment complications. We are developing a new Precision Infection Management (PIM) strategy for achieving these objectives. Our approach links the complete proteomic, metabolomic, and genomic sequences of 50,000 microbial isolates to birth-to-death medical records and integrates microbial risk-factors into a hand-held smartphone app for clinicians.50,000 isolates spanning two decades of clinical diagnostic work in southern Alberta are being cultured in 96-well format and DNA, protein, and metabolites are being extracted using an automated workflow. Full genome sequences are being collected by the Broad Institute, quantitative proteomics analyses are being acquired using a TMT11plex workflow, and metabolomics data are being acquired using our metabolic preference assay. De-identified patient data have been collected from Alberta Health Services and these records are being linked to each clinical isolate to enable microbe/clinical outcome association studies. All data are being stored on a new secure data hub, ResistanceDB, which supports complex multiomics data mining, machine learning, and microbial risk assignment. We are currently establishing the microbiology, analytical, and informatics pipelines necessary to support the comprehensive analyses of 50,000 microbial isolates. We have recently launched our pilot ResistanceDB hub, and have collected genomics, proteomics, and metabolomics data on a pilot set of 1,000 clinically-linked microbial isolates. We have established automated data capture and archival systems to collect data from each of the 'omics pipelines and have invested significant time in evaluating practical methods for analyzing thousands of microbial samples. We are currently using our computational resources to survey the current state of microbial populations and document their evolution over the last decade. Our preliminary analyses show a dramatic rise in the prevalence of resistant organisms over this time. We have developed several new visualization tools for projecting microbial population-level changes over time and we are currently working to understand how these microbial dynamics have affected patient outcome. Our quantitative proteomics methods are currently capturing over 1,000 microbial proteins, including most of the know virulence factors and our metabolomics assay is capturing more than 250 metabolites from a transect of central carbon metabolism. We are currently transitioning 
into scale-up of the program and are building the informatics tools that will ultimately allow our microbial risk scores to be made available to clinicians via a smart-phone enabled app. The primary objective of this work is to enable the precision management of infections using isolate-specific virulence data. This PIM approach will inform clinical decision-making and infection management practices in point-of-care settings resulting in, (1) a reduction in the number of people who develop life-threatening infections, (2) a reduction in the number of side effects that result from over-treating benign infections, and (3), an extended service-life for our existing antibiotics by dramatically reducing the over-use of these drugs. We introduce a new Precision Infection Management (PIM) strategy for titrating clinical care according to the risks posed by each individual infection.

Disclosure No significant relationships.

\section{S15.4 COMBATTING HIV WITH NANOMATERIALS}

Kim A Woodrow*. University of Washington, Bioengineering, Seattle, USA

\subsection{6/sextrans-2019-sti.72}

The delivery of drug combinations is a paradigm for treatment of cancer, HIV/AIDS and drug resistant bacterial infections. My laboratory is interested in the application of engineered biomaterials to control the spatial and temporal delivery of a combination of agents (small molecules, biologics, conjugates). Strategies to combine chemically incompatible agents may facilitate the discovery of unique drug-drug activities, particularly unexplored combination drug synergy. In this presentation, I will summarize our efforts to develop polymeric delivery systems for the combination delivery of antiretroviral (ARV) drugs in HIV prevention, treatment and cure. We have developed polymeric particulate and fiber carrier systems for delivering ARV drug combinations. The flexibility to design the nanoarchitecture of these polymeric carriers, combined with the versatility of drugs that can be encapsulated for controlled release, motivate the use of these systems for topical, injectable or oral delivery of combination agents.

\section{S16.1 - ANATOMICAL SITES OF INFECTION: BIOMEDICAL, MODELING, BEHAVIORAL, AND PROGRAMMATIC CONSIDERATIONS FOR STI PREVENTION}

\section{Wednesday, July 17, 2019 10:45 AM - 12:15 PM}

\section{S16.1 ANATOMICAL SITES OF INFECTION - BIOMEDICAL CONSIDERATIONS FOR STI PREVENTION}

Jane Hocking*. University of Melbourne, Melbourne School of Population and Global Health, Carlton, Australia

10.1136/sextrans-2019-sti.73

Extragenital (anorectal and oropharyngeal) sexually transmitted infections (STIs), particularly chlamydia and gonorrhoea, are highly prevalent among men who have sex with men (MSM), but are now an increasing concern among heterosexual men and women with calls for anorectal testing in women. Further, there is ongoing debate about the role of oropharyngeal STIs driving transmission, particularly among MSM and about anorectal chlamydia in women causing urogenital infection via auto-inoculation. These issues highlight that site of infection is an important issue. Treatment efficacy can vary considerably by site of infection - for example, treatment efficacy is lower for oropharyngeal gonorrhoea and anorectal chlamydia with some treatments. Factors related to the pharmacokinetic properties of the drug can affect its efficacy at different anatomic sites including its tissue distribution, protein binding and half-life. Factors related to the individual including the $\mathrm{pH}$ of the local tissue environment, immune response, drug side-effects and sexual practices can affect treatment efficacy. Finally, factors related to the microorganism itself such as organism load and antimicrobial resistance can also impact on treatment efficacy. These factors should play a role in guiding treatment guidelines as it is possible that treatment regimens need to vary by site of infection. The use of mouthwash and doxycycline prophylaxis have been raised as potential biomedical interventions to reduce STI transmission, although ongoing concern about antimicrobial stewardship threatens the widespread use of doxycycline. We also need to understand the natural history of extra-genital STIs, particularly in women where the importance of anorectal infections and whether they play an important role in inoculating and causing persistent urogenital infection is not well understood. This presentation will discuss the importance of the site of infection particularly when considering treatment options and the possible role of biomedical interventions to prevent infection.

Disclosure No significant relationships.

\section{S16.2 MODELING CONSIDERATIONS RELATED TO MULTI-SITE INFECTION}

Ian Spicknall*. Centers for Disease Control and Prevention, Atlanta, USA

\subsection{6/sextrans-2019-sti.74}

Background Gonorrhea may infect the urethra, rectum, and oropharynx in men. It may only be acquired when there is contact between infected and uninfected anatomical sites. Seven plausible routes of MSM transmission have been proposed: urethra-to-rectum, rectum-to-urethra, urethra-to-oropharynx, rectum-to-oropharynx, oropharynx-to-urethra, oropharynx-to-rectum, and oropharynx-to-oropharynx. We characterize the uncertainty and potential importance of transmission from each anatomical site using a deterministic compartmental mathematical model.

Methods We developed a model of site-specific gonococcal infection, where individuals are infected at zero, one, two, or all three sites. Sexual behavior and infection duration parameters were fixed similar to recent analyses. Markov Chain Monte Carlo methods were used to sample the posterior distribution of transmission probabilities that were consistent with site-specific prevalence in American MSM populations under specific scenarios. Scenarios were defined by whether transmission routes may or may not transmit by constraining specific transmission probabilities to zero rather than fitting them. 